\title{
Cosmological Solution from D-brane motion in NS5-Branes background
}

\author{
Hossein Yavartanoo ${ }^{1}$ \\ Institute for Studies in Theoretical Physics and Mathematics (IPM) \\ P.O. Box 19395-5531, Tehran, Iran
}

\begin{abstract}
We study dynamics of a D3-brane propagating in the vicinity of $\mathrm{k}$ coincident NS5 branes. We show that when $g_{s}$ is small, there exists a regime in which dynamics of the D-brane is governed by Dirac-Born-Infeld action while higher order derivative in the expansion can not be neglected. This leads to a restriction on how fast scalar field may roll. We analyze the motion of a rolling scalar field in this regime, and extend the analysis to cosmological systems obtained by coupling this type of field theory to four dimensional gravity. It also leads to some FRW cosmologies, some of which are related to those obtained with tachyon matter.
\end{abstract}

\footnotetext{
${ }^{1}$ yavar@ipm.ir
} 


\section{Introduction}

The dynamics of branes in string theory is an important subject, which we hope to provide clues about the fundamental description of the string theory. The time dependent nature of the solution is challenging and includes many puzzling issues, while important for many applications in cosmological problems. One of the most well studied subject is the time evolution of unstable D-branes in flat backgrounds, that is the problem of rolling tachyons [1]-[8](see also $[9,10])$.

In studies of D-branes in string theory one often finds open string tachyon in the lowest lying open string states. This happens in bosonic and superstring theory as well, which admit time dependent solutions describing the rolling of the tachyon away from the maximum of the potential.

The rolling of tachyon towards a minimum of the potential in some cases can be understood exactly in the context of classical open string theory. Thus, in general the study of tachyon condensation provides useful insights on the time-dependent solutions in string theory. These solutions involve the tachyon field, in which the tachyon rolls away from the maximum of the potential, also display novel features. They are interesting solutions, both in describing the early universe and in studying various dynamical issues.

A more ambitious problem may be time dependent string or brane dynamics in curved backgrounds. A significant example is studying instability of (BPS) D-brane, associated with the presence of NS5-brane in its vicinity. Recently Kutasov, using the Dirac-Born-Infeld action, has studied the time evolution of D-branes near the stack of NS5-branes(for earlier works see also [16]), emphasizing the formal resemblance to the rolling tachyon problem. As it was shown this instability is closely related to the tachyon condensation problem and one can say a lot about features of time dependent solutions on it.

Indeed a configuration of parallel NS5-branes and D3-brane breaks supersymmetry completely, since the NS5-brane and D-brane preserve different halves of the supersymmetry of type II string theory. The D-brane carries RR charge, but this charge can leak from D-brane to the NS5-brane. If we place D-brane at a finite distance from a stack of NS5-branes, it will experience an attractive force and start moving towards the fivebranes. In this paper we will studying some cosmological consequences which arise from D-brane rolling down the throats of NS5-branes.

There are several cosmological models which are based on the dynamics of un-

stable branes in string theory (see e.g. [17]-[34] and references therein). Brane cosmology assumes that our universe starts out with branes embedded in higher dimensional spacetime, either stable or unstable. In these scenarios a natural can- 
didate for inflaton is the brane mode, whose expectation value describes the inter brane separation. The dynamics of inflation is therefore governed by the interaction between D-branes. The potential which gives rise to the inflation during the slow roll epoch is well studied in string theory when the branes are far apart. When the brane are sufficiently close to each other, tachyons develop. The slow change in inter-brane separation is followed by quick roll down the tachyonic direction.

In [35] a "D-ecelerations" mechanism for slowing scalar field motion was identified in the context of strongly 't Hooft coupled conformal field theories. The slow motion of the scaler is understood in simple way from gravity side of the AdS/CFT correspondence which provides the effective description of the system. In terms of the effective action this behavior occurs due to the higher derivative terms encoded in Dirac-Born-Infeld (DBI) action. When the effective field theory is subsequently coupled to gravity, this mechanism leads to inflation in much steeper potential than are allowed in standard weakly coupled slow roll inflation.

In this paper, we use this idea to study cosmological solution arising from moving a D-brane in the vicinity of NS5-branes. We show that there exists a regime, in which the string coupling at the location of the D-brane is small and then we can use DBI action to study the dynamics of D-brane in this background while higher derivative terms in DBI action can not be neglected. The non-analytic behavior of the square-root of DBI action in this regime gives rise to a speed limit restriction on how fast the scalar field on the brane (inflaton) can roll. We study this system when the effective field theory is coupled to gravity and we show that this mechanism leads to inflation for some potentials.

The paper is organized as follows. We start in section 2 by reviewing the near horizon geometry on NS5-branes (the CHS geometry) and DBI action for D-brane in this background. In section 3, we couple this system to gravity and study cosmologies arising from our low energy effective action. In section 5 we try to solve the equation of motion for scale factor for some different potentials, and we show that there exists an inflationary solution in some cases. 


\section{D-brane effective action in the NS5-brane back- ground}

Consider a stack of $\mathrm{k}$ coincident NS5-branes in type II string theory, stretched in directions $\left(x^{1}, \ldots, x^{5}\right)$ and localized in $\vec{x}=\left(x^{6}, x^{7}, x^{8}, x^{9}\right)$. The direction along the worldvolume of the fivebranes will be denoted by $x^{\mu}, \mu=0,1,2, \ldots 5$; those transverse to the branes will be labelled by $x^{m}, m=6,7,8,9$. The background fields are given by CHS solution [36].

The metric, dilaton and NS B-field are

$$
\begin{aligned}
d s^{2} & =d x_{\mu} d x^{\mu}+H(r)\left(d r^{2}+r^{2} d \Omega_{3}^{2}\right) \\
e^{2 \Phi} & =g_{s}^{2} H(r) \\
H_{i j k} & =-\epsilon_{i j k}^{l} \partial_{l} \Phi
\end{aligned}
$$

Here $r$ is the radial direction perpendicular to NS5-branes and $d \Omega_{3}^{2}$ is the metric on $S^{3} . H_{i j k}$ is the field strength of the $N S$ B-field and $H(r)$ is a harmonic function on the transverse space

$$
H(r)=1+\frac{k \alpha^{\prime}}{r^{2}}
$$

We are interested is the dynamics of BPS Dp-brane in this background. The Dbrane is parallel to the fivebrane, i.e. it is extended in some or all of the fivebrane worldvolume directions $x^{\mu}$ and point like in the directions transverse to the fivebranes $\left(x^{6}, x^{7}, x^{8}, x^{9}\right)$. Without loss of generality, we can take the worldvolume of the Dp-brane to fill the directions $\left(x^{0}, x^{1}, \ldots, x^{p}\right)$. We will label worldvolume of the Dp-brane by $x^{\mu}$ as well, but one should bear in mind that here the index $\mu$ only runs over $\mu=0,1,2, \ldots p$, with $p \leq 5$.

Although the D-brane in question is BPS, in the presence of the fivebranes it is unstable. Indeed, a configuration of parallel NS5-branes and Dp-brane breaks supersymmetry completely, since the fivebrane and D-brane preserve different halves of the supersymmetry of type II string theory. The Dp-brane carries RR charge, but this charge can leak from D-brane to fivebranes.

If we place a BPS Dp-brane at finite distance from a stack of NS5-branes, it will experience an attractive force, and thus start moving towards the fivebranes. The mass per unit volume of a D-brane in string units is $1 / g_{s}$, where $g_{s}$ is the string coupling. While that of a NS5 brane goes like $1 / g_{s}^{2}$. Therefore at weak string coupling NS5 branes are much heavier than D-branes. We can take the NS5 brane to be static and study the motion of a D-brane in this background. 
Consider a Dp-brane in this background, we label the worldvolume of a D-brane by $\xi^{\mu}, \mu=0,1,2, \ldots, p$, and use reparametrization invariance on the world-volume of the D-brane to set $\xi^{\mu}=x^{\mu}$. The position of the D-brane in the transverse directions $\left(x^{6}, . ., x^{9}\right)$ gives rise to scaler fields on its world-volume, $\left(X^{6}\left(\xi_{\mu}\right), \ldots, X^{9}\left(\xi_{\mu}\right)\right)$. The effective action on the world-volume on the D-brane is the DBI action [37]

$$
S=-T_{p} \int d^{p+1} \xi e^{-\left(\Phi-\Phi_{0}\right)} \sqrt{-\operatorname{det}\left(G_{\mu \nu}+B_{\mu \nu}\right)} .
$$

$T_{p}$ is the tension of the Dp-brane and $G_{\mu \nu}, B_{\mu \nu}$ are induced metric and B-field on the D-brane. They are related to the metric and B-field in ten dimension by

$$
\begin{aligned}
G_{\mu \nu} & =\frac{\partial X^{A}}{\partial \xi^{\mu}} \frac{\partial X^{B}}{\partial \xi^{\nu}} G_{A B}(x) \\
B_{\mu \nu} & =\frac{\partial X^{A}}{\partial \xi^{\mu}} \frac{\partial X^{B}}{\partial \xi^{\nu}} B_{A B}(x) .
\end{aligned}
$$

The indices $A$ and $B$ run over the whole ten dimensional spacetime.

An interesting special case of the action (3) is obtained when we restrict ourselves to purely radial fluctuations of the D-brane in transverse space $\mathbb{R}^{4}$ labelled by $\vec{x}$. For such fluctuations the only excited field on brane is $r\left(\xi_{\mu}\right)$ and the angular variables remains fixed at their initial values. This restriction on the radial motion is consistent since, for coincident NS5-branes, the background (1) is $S O(4)$ invariant, and the D-brane experiences a central force pulling it towards the origin.

Since the B-field (1) is in the angular directions, and the angular degrees of freedom are not excited, the induced B-field in (3) vanishes. The DBI action in this case is thus given by

$$
S=-T_{p} \int d^{p+1} x \frac{1}{\sqrt{H}} \sqrt{1+H \partial_{\mu} r \partial^{\mu} r}
$$

As it was mentioned in [14], the form of the action (5) is very reminiscent of the DBI action of the tachyon in open string models [1]. After defining the variable $T$ the "tachyon field" by

$$
T=\sqrt{k \alpha^{\prime}+r^{2}}+\frac{1}{2} \sqrt{k \alpha^{\prime}} \ln \frac{\sqrt{k \alpha^{\prime}+r^{2}}-\sqrt{k \alpha^{\prime}}}{\sqrt{k \alpha^{\prime}+r^{2}}+\sqrt{k \alpha^{\prime}}}
$$

the action (3) has the similar form of the tachyon action in open string models

$$
S_{\text {tach }}=-\int d^{p+1} x V(T) \sqrt{1+\partial_{\mu} T \partial^{\mu} T},
$$

where the asymptotic behavior of potential $V(T)$ is given by

$$
\frac{1}{T_{p}} V(T) \simeq \begin{cases}\exp \frac{T}{\sqrt{k \alpha^{\prime}}} & T \rightarrow-\infty \\ 1-\frac{k \alpha^{\prime}}{2 T^{2}} & T \rightarrow \infty\end{cases}
$$


Note that even when the D-brane is very close to the fivebranes, there is no perturbative string tachyon here, because a fundamental string can not stretch between the D-brane and the NS5-branes.

In this case the "tachyon field" $T$ acquires a geometrical meaning and is related via (6) to the distance between the D-brane and the fivebranes. From the asymptotic behavior of the potential (8) when $T \rightarrow-\infty$ we recognize that the potential goes exponentially to zero. This is precisely the behavior exhibited at late times by the tachyon potential relevant for rolling tachyon solution. This behavior of the potential leads to the absence of plane wave solution around the minimum of the potential at $T \rightarrow-\infty$, and to the exponential decrease of the pressure at late times [4]. For unstable D-branes a similar equation of state is taken to signal the decay of the D-brane into the closed string radiation, see e.g.[13, 12, 11]. In [14] it was proposed that in the case of D-brane in fivebranes background at late times the D-brane decays into modes propagating in the fivebrane throat.

In the next sections we restrict our attention to the case of $p=3$, and we will try to investigate what types of cosmologies arise from D3-branes rolling down the throat of NS5 brane.

\section{Coupling with gravity}

In [35], motivated by the behavior of rolling scalar fields in the strong coupling limit of theories, a new mechanism for slow roll inflation was analyzed using the AdS/CFT correspondence. The non-analytic behavior of the square-root in the DBI action gives rise to a speed limit restricting how fast the scalar field may roll. This is nothing but the Einstein's causal speed limit in the holographic dimension, and is the crucial bit of new physics in our inflationary mechanism.

Motivated by this scenario, here we want to generalize the action to incorporate the effects of coupling to four dimensional gravity as well as to the other sectors suppressed by higher dimension operators that may arise in the corresponding string compactifications. We can also ask what is the low-energy effective action of (5) when the gauge theory is coupled to four dimensional gravity introducing by dynamical background metric $g_{\mu \nu}$. A simple possible generalization can be given by adding a potential term $V$ to the action that arise when the system is coupled to four dimensional gravity and other sectors involved in a full string compactification. Four dimensional covariantization of (5) involving the potential $V$ is

$$
S=-\frac{1}{\alpha^{\prime} g_{Y M}^{2}} \int d^{4} x \sqrt{-g}\left(\frac{1}{\sqrt{f(\phi)}} \sqrt{1+f(\phi) g^{\mu \nu} \partial_{\mu} \phi \partial_{\nu} \phi}+V(\phi)\right)
$$

In the expression above we have use field theory variable which is defined by $\phi=$ 
$r / \alpha^{\prime}$, we have also defined $f(\phi)=\alpha^{\prime 2}+\lambda^{2} / \phi^{2}$ and $\lambda^{2}=\alpha^{\prime} k$.

Now we can investigate some cosmological features of this system, by studying FRW cosmologies solutions which follow from (9). We consider only flat cosmologies,

$$
d s^{2}=-d t^{2}+a(t)^{2} d x^{2} .
$$

Since spatially inhomogeneous terms are redshifted away during inflation, we consider the scalar field ansatz $\phi=\phi(t)$. Here we also ignore the effects of conformal coupling $\mathcal{R} \phi^{2}$, we will comment on the reliability of this consideration further below and we will show that its effects are self consistently negligible in our solutions. With this ansatz the equation of motion can be expressed by first defining $\gamma$ [35] analogous of the Lorentz contraction factor in special relativity

$$
\gamma=\frac{1}{\sqrt{1-f(\phi) \dot{\phi}^{2}}}
$$

The energy density $\rho$ and pressure $p$ following from (9) are given by

$$
\begin{aligned}
& \rho=\frac{\gamma}{\sqrt{f}}+V \\
& p=-\frac{1}{\gamma \sqrt{f}}-V,
\end{aligned}
$$

These definitions do not include overall coefficient of $1 /\left(\alpha^{\prime} g_{Y M}^{2}\right)$ which instead combines with $M_{p}$ in the Einstein equations so that the scale $\left(M_{p} \sqrt{g_{s}}\right)=M_{p} g_{Y M}$ appears in all equations. The Friedmann equations read,

$$
\begin{aligned}
3 H^{2} & =\frac{1}{g_{s} \alpha^{\prime} M_{p}^{2}} \rho \\
2 \frac{\ddot{a}}{a}+H^{2} & =-\frac{1}{g_{s} \alpha^{\prime} M_{p}^{2}} p
\end{aligned}
$$

Here $H=\dot{a} / a$ is the usual Hubble parameter. We will try to find solutions in which the brane is asymptotic to the speed of light at late times in the gravity side background. This means that the quantity $\gamma$ grows when $t$ gets large, and the behavior of $\rho$ will be substantially different from the usual case of $\rho=\frac{1}{2} \dot{\phi}^{2}+V$, in which reduces at small proper velocity. Firstly, we try to write the Friedmann equations (13) in a more suitable form. In fact they can be integrated at once. It is referred to the "Hamilton-Jacobi" formalism [38]. Another perspective can be obtained by viewing the resulting cosmology as a Wick rotation of a BPS domain wall; the first order Friedmann equations are related to the Bogolomoln'yi equations derived in [39].

We can derive the Hamilton-Jacobi formalism equations by viewing the scalar field $\phi$ as a time variable. In practice, this means that we can consider $H=H(\phi)$ 
with $\phi=\phi(t)$. It is important to notice that this assumption puts a limit on the dynamics, since it considers $\phi$ as a monotonic function, and $H$ is a single valued function of $\phi$ and therefore it restricts us to non-oscillatory behavior in $\phi$ [14].

If we take time derivative of the first Friedmann equation and then using the second equation, we find

$$
6 H H^{\prime} \dot{\phi}=-\frac{1}{g_{s} \alpha^{\prime} M_{p}^{2}} 3 H \gamma \sqrt{f} \dot{\phi}^{2},
$$

which can be simply solved by using (11) and it results,

$$
\dot{\phi}=\frac{-2 H^{\prime}}{\sqrt{f /\left(g_{s}^{2} \alpha^{\prime 2} M_{p}^{4}\right)+4 f H^{\prime 2}}} .
$$

Finally after substituting into the first Friedmann equation and using (12) we get an expression for the potential $V(\phi)$ in terms of Hubble parameter $H(\phi)$,

$$
V=3 g_{s} \alpha^{\prime} M_{p}^{2} H^{2}-\sqrt{\left(1+4 g_{s}^{2} \alpha^{\prime 2} M_{p}^{4} H^{\prime 2}\right) / f}
$$

Now if we are able to solve (16) for a given potential $V$, we can plug the solution into the expression (15) to find $\phi(t)$, and it can be used to find the scale factor as a function of time.

Before trying to solve this equation, let us discuss a little about the range of reliability of the solutions. The DBI action is reliable to describe the dynamics of D-brane, when the string coupling at the location of the brane is small, i.e. one must have $\exp (\Phi) \ll 1$. Since from equation (1) the dilaton grows without bound as one goes down the throat of fivebranes, it gives us a lower bound on $\phi$ and also an upper bound on $g_{s}$

$$
g_{s} \ll 1 \quad \text { and } \quad \lambda g_{s} / \alpha^{\prime} \ll \phi .
$$

In this limit, the DBI action is a good description for D-brane dynamics, however there is also an upper limit for $\phi$. In fact we are entitled to take $\phi$ as large as we wish and for $\phi \gg M_{p}$, one finds standard slow roll expansion of chaotic inflation. However, as a result of discussion in [35], models with super Planckian VEVs suffer from destabilization from a slew of quantum corrections involving for example gravitational conformal couplings $\mathcal{R} \phi^{2}$ [17]. To avoid this, we shall instead restrict ourselves to subPlanckian VEVs, $\phi \ll M_{p}$. This allows us to avoid destabilizing effects, circumventing some of the difficulties involved in placing inflation within a string comactification $[17,40,41]$. Combining these two condition we find

$$
g_{s} k / \lambda \ll \phi \ll M_{p}
$$

In the next section we shall try to solve equation (16) when $\phi$ lies in (18) regime, for some relevant potentials. 


\section{Cosmological Solution}

As mentioned before, we can consider the coupling of the system to four dimensional gravity and also other sectors involved in full string compactification. This whole could be described by a potential $V(\phi)$ in action (9). Consider power series expansion of the potential $V(\phi)$ as

$$
V=V_{0}+V_{2} \phi^{2}+V_{4} \phi^{4} \ldots
$$

The most important term in the Lagrangian at low energy is the hard cosmological constant $V_{0}$. As we will see below, giving a finite non-zero value to $V_{0}$, dramatically affects the solutions of (16). This term gets contribution in principle from all sectors of the system. In effective field theory ( and approximately in string theory by using the Bousso-Polchinski mechanism [42]) we may turn this (close) to the value of interest for a given application. We will make use of this freedom in our analysis.

case $V_{0} \neq 0$

Let us first consider the interesting case that will give rise to inflation. We suppose that $V_{0} \neq 0$ so that this term dominates when $\phi$ is small. When $\phi$ lies in the regime (18), the solution of equation (16) can be expanded as

$$
H(T)=h_{0}+h_{1} \phi+h_{2} \phi^{2}+\ldots
$$

where coefficients $h_{i}$ 's are

$$
h_{0}=\sqrt{\frac{V_{0}}{3 g_{s} \alpha^{\prime} M_{p}^{2}}}, \quad h_{1}=\frac{1}{\sqrt{12 \alpha^{\prime} g_{s} M_{p}^{2} \lambda^{2} V_{0}-4 g_{s}^{2} \alpha^{\prime 2} M_{p}^{4}}}, \quad \ldots
$$

In expression (20) we can see that $V_{2 i}$ dependence of $h_{j}$ appears for $j \geq 2 i$. Substituting this into the first Friedmann equations, we find can $\phi(t)$ at late times and then from (20) we read scale factor as a function of time.

The condition for acceleration $(\ddot{a}>0)$ and D-cceleration $(\gamma>1)$ in terms of the potential $V$ can be written as follows ${ }^{2}[35]$

$$
\begin{aligned}
& \frac{V^{3 / 2}}{M_{p} V^{\prime} \phi} \sqrt{\frac{3 k}{g_{s}}} \gg 1 \\
& \frac{g_{s}}{3 k V} \lambda^{2} M_{p}^{2} V^{\prime 2} \gg 1
\end{aligned}
$$

These are to be contrasted with the usual slow roll conditions $\left(V^{\prime} / V\right)^{2} M_{p}^{2} \ll 1$ and $V^{\prime \prime} / V M_{p}^{2} \ll 1$.

\footnotetext{
${ }^{2}$ I would like to thank E. Silverstein for her useful comments and bringing my attention to this point.
} 
For example for a quadratic potential of the form

$$
V=V_{0}+V_{2} \phi^{2},
$$

these conditions can be satisfied for given $V_{0} \sim \mathcal{O}\left(g_{s} M_{p}^{2}\right)$ and $V_{2} \sim \mathcal{O}\left(1 / g_{s}\right)$. In this case it is easy to see that the proper acceleration is much smaller than string scale. This condition is necessary for self-consistency of our solution.

The number of e-foldings is given by

$$
N_{e} \sim \int_{\phi_{f}}^{\phi_{i}} \frac{d \phi}{M_{p}} \sqrt{\frac{k V}{3 g_{s} \phi^{2}}}
$$

For the potential (24), it leads to $N_{e} \sim \sqrt{k} / g_{s}$, which gives us sufficient large number in the small $g_{s}$ limit.

case $V_{0}=0$

Let us now suppose that the constant term in the potential is vanishing. As we will see in this case the solution of equation (16) has completely different behavior in comparison with the the previous case with $V_{0} \neq 0$. This comes from the fact that when $\phi$ lies in the regime (18), the first term in the Lagrangian (9) will dominate and then we expect a different behavior for the scale factor. Firstly, we want to re-write the equation (16) in a more tractable form. In fact in this case, it is natural to write the equation in terms of the "tachyon field", which in the limit (18) is given by

$$
T \simeq \lambda \ln \frac{\phi}{\lambda}
$$

Now in terms of "tachyon filed", equation (16) reads as

$$
V=3 g_{s} M_{p}^{2} H^{2}-\sqrt{e^{2 T / \lambda}+4 g_{s}^{2} \alpha^{2} M_{p}^{4} H^{\prime 2}},
$$

while the condition (18) restricts $T$ to the large negative values. Now the equation (27) can be solved approximately by

$$
\begin{aligned}
H(T)= & -\frac{2}{3 T}-\frac{3 \lambda}{32 g_{s}^{2} \alpha^{\prime 2} M_{p}^{4}} T^{2} e^{2 T / \lambda}+\frac{3 \lambda^{2}}{16 g_{s}^{2} \alpha^{\prime 2} M_{p}^{4}} T e^{2 T / \lambda} \\
& +\frac{\left(9+8 V_{2} g_{s} \alpha^{\prime} M_{p}^{2}\right) \lambda^{3}}{32 g_{s}^{2} \alpha^{\prime 2} M_{p}^{4}} e^{2 T / \lambda}+\ldots
\end{aligned}
$$

substituting this into the expression (15), we get the equation of motion for $T$ at late times

$$
\dot{T}=-1+\frac{9 T^{4}}{32 g_{s}^{2} M_{p}^{4}} e^{2 T / \lambda}+\ldots
$$


Then the asymptotic behavior of $\mathrm{T}(\mathrm{t})$ for late times reads as

$$
T(t)=-t+\frac{9}{64 \lambda g_{s}^{2} \alpha^{\prime 2} M_{p}^{4}} t^{4} e^{-2 t / \lambda}+\ldots
$$

and we can determine the behavior of the Hubble constant in this regime

$$
H(t)=\frac{2}{3 t}+\frac{3}{32 g_{s}^{2} M_{p}^{4} \lambda} t^{4} e^{-2 t / \lambda}+\ldots
$$

which looks like the matter dominant solution of the Freidmann equation, when the matter is a noninteractive dust. In fact for any potential with $V_{0}=0$ and nonvanishing higher order terms, the rolling scalar field looks to have equation of state $\omega \approx 0$; which is a kinetic dust.

\section{Conclusion}

In this paper we have studied some cosmological solutions which arise from D3brane rolling down the throats of $\mathrm{k}$ coincident NS5-branes. We started by coupling this system to four dimensional gravity and studied possible FRW cosmological solutions. We have shown that for various potentials $V$, which describe the coupling of the system to four dimensional gravity (and also other sectors involved in a full string compactification), different solutions appear. If the potential has a constant non-zero value in its expansion we have inflationary solution for special range of parameters. We also found that for $V_{0}=0$ the late times behavior of the system is very similar to dynamics of tachyon in non-BPS branes. In this case we found the late times behavior of scalar field is similar to non-interactive dust. In the context of tachyon condensation, the pressureless fluid behavior at late times is usually a signal of the instability of the D-brane. This has been argued to provide a dual description of the closed string radiation that D-brane decays into. As it was mentioned in [14] it is natural to assume that a similar interpretation can be made here as well, with D-branes decaying into modes living on the fivebrane.

For the inflationary solution that we have found, it is interesting to analyze the density perturbations resulting from inflationary solution found in [43]-[48]. The DBI Lagrangian is proportional to $\sqrt{1-v_{p}^{2}}$, where $v_{p}=\lambda \dot{\phi} / \phi$ is the gravity side proper velocity of the brane probe whose position collective coordinate is the inflaton $\phi$. The inflationary solution involves a proper velocity approach to speed of light as $\phi$ approaches small values. Expanding the action in fluctuations of $\phi$ involves expanding the square root in the DBI Lagrangian, which produces powers of $\gamma=1 / \sqrt{1-v_{p}^{2}}$ accompanying powers of the fluctuations of the inflation. Since $\gamma$ is relatively large, this may produce a large contribution to non-Gaussianities. This issue is under investigation [49]. 
It is also interesting to study D-brane dynamics in the deformed NS5-brane backgrounds and cosmologies arising from that[50].

\section{6 acknowledgments}

I would like to thank S. Alexander, M.Alishahiha, A. Imaanpur, S. Parvizi, S. Sheikh-Jabbari E. Silverstein for useful and interesting discussion.

\section{References}

[1] A. Sen, "Rolling tachyon" JHEP 0204, 048 (2002) [arXiv:hep-th/0203211].

[2] A. Sen, "Supersymmetric world-volume action for non-BPS D-branes," JHEP 9910, 008 (1999) [arXiv:hep-th/9909062].

[3] A. Sen, Phys. Rev. D 68, 066008 (2003) [arXiv:hep-th/0303057].

[4] A. Sen, "Tachyon matter" JHEP 0207, 065 (2002) [arXiv:hep-th/0203265].

[5] A. Sen, "Field theory of tachyon matter," Mod. Phys. Lett. A 17, 1797 (2002) [arXiv:hep-th/0204143].

[6] A. Sen, "Open-closed duality: Lessons from matrix model' arXiv:hepth/0308068.

[7] A. Sen, "Open-closed duality at tree level," Phys. Rev. Lett. 91, 181601 (2003) [arXiv:hep-th/0306137].

[8] A. Sen "Remarks on tachyon driven cosmology," arXiv:hep-th/0312153.

[9] M. Gutperle and A. Strominger, "Spacelike branes," JHEP 0204, 018 (2002) [arXiv:hep-th/0202210].

[10] F. Larsen, A. Naqvi and S. Terashima, "Rolling tachyons and decaying branes," JHEP 0302, 039 (2003) [arXiv:hep-th/0212248].

[11] T. Okuda and S. Sugimoto, "Coupling of rolling tachyon to closed strings," Nucl. Phys. B 647, 101 (2002) [arXiv:hep-th/0208196].

[12] N. Lambert, H. Liu and J. Maldacena, "Closed strings from decaying Dbranes", arXiv:hep-th/0303139.

[13] D. Gaiotto, N. Itzhaki and L. Rastelli, "Closed strings as imaginary D-branes," arXiv:hep-th/0304192.

[14] D. Kutasov, "D-brane dynamics near NS5-branes," arXiv:hep-th/0405058. 
[15] Y. Nakayama, Y. Sugawara, H. Takayanagi "Boundary States for the Rolling D-branes," arXiv:hep-th/0406173

[16] S. Elitzur, A. Giveon, D. Kutasov, E. Rabinovici and G. Sarkissian, "D-branes in the background of NS fivebranes," JHEP 0008, 046 (2000) [arXiv:hepth/0005052]. O. Pelc, "On the quantization constraints for a D3 brane in the geometry of NS5 branes," JHEP 0008, 030 (2000) [arXiv:hep-th/0007100]. S. Ribault, "D3-branes in NS5-branes backgrounds," hep-th/0301092, JHEP 0302, 044 (2003).

[17] S. Kachru, R. Kallosh, A. Linde, J. Maldacena, L. McAllister and S. P. Trivedi, "Towards inflation in string theory" arXiv:hep-th/0308055.

[18] G. R. Dvali and S. H. H. Tye, Phys. Lett. B 450, 72 (1999) [arXiv:hep$\mathrm{ph} / 9812483]$.

[19] A. Maloney, A. Strominger and X. Yin, "S-brane thermodynamics" arXiv:hepth $/ 0302146$.

[20] G. W. Gibbons, "Cosmological evolution of the rolling tachyon" Phys. Lett. B 537, 1 (2002) [arXiv:hep-th/0204008].

[21] G. Shiu, S. H. H. Tye and I. Wasserman, "Rolling tachyon in brane world cosmology from superstring field theory," Phys. Rev. D 67, 083517 (2003) [arXiv:hep-th/0207119].

[22] A. Feinstein, "Power-law inflation from the rolling tachyon", Phys. Rev. D 66, 063511 (2002) [arXiv:hep-th/0204140].

T. Padmanabhan, "Accelerated expansion of the universe driven by tachyonic matter" Phys. Rev. D 66, 021301 (2002) [arXiv:hep-th/0204150].

L. R. Abramo and F. Finelli, "Cosmological dynamics of the tachyon with an inverse power-law potential", arXiv:astro-ph/0307208.

[23] T. Banks, M. Berkooz, S. H. Shenker, G. W. Moore and P. J. Steinhardt, "Modular cosmology" Phys. Rev. D 52, 3548 (1995) [arXiv:hep-th/9503114].

[24] T. Banks, W. Fischler and L. Motl, "Dualities versus singularities", JHEP 9901, 019 (1999) [arXiv:hep-th/9811194].

[25] S. H. S. Alexander, Phys. Rev. D 65, 023507 (2002) [arXiv:hep-th/0105032].

[26] F. Quevedo, "Lectures on string / brane cosmology" Class. Quant. Grav. 19, 5721 (2002) [arXiv:hep-th/0210292].

[27] C. Bachas, "D-brane dynamics", Phys. Lett. B 374, 37 (1996) [arXiv:hepth/9511043]. 
[28] M. R. Douglas, D. Kabat, P. Pouliot and S. H. Shenker, "D-branes and short distances in string theory", Nucl. Phys. B 485, 85 (1997) [arXiv:hepth/9608024].

[29] C. P. Burgess, P. Martineau, F. Quevedo and R. Rabadan, "Branonium", JHEP 0306, 037 (2003) [arXiv:hep-th/0303170].

[30] M. Brandle and A. Lukas, "Flop transitions in M-theory cosmology", Phys. Rev. D 68, 024030 (2003) [arXiv:hep-th/0212263].

[31] S. Alexander, R. Brandenberger and M. Rozali, arXiv:hep-th/0302160.

[32] L. Jarv, T. Mohaupt and F. Saueressig, "M-theory cosmologies from singular Calabi-Yau compactifications", arXiv:hep-th/0310174.

[33] G. Calcagni, "Slow-roll parameters in braneworld cosmologies," Phys. Rev. D 69, 103508 (2004) [arXiv:hep-ph/0402126].

[34] A. Buchel and A. Ghodsi, arXiv:hep-th/0404151.

[35] E. Silverstein and D. Tong, "Scalar speed limits and cosmology: Acceleration from D-cceleration," arXiv:hep-th/0310221. M. Alishahiha, E. Silverstein and D. Tong, "DBI in the sky," arXiv:hep-th/0404084.

[36] C. G. . Callan, J. A. Harvey and A. Strominger, "Supersymmetric string solitons," arXiv:hep-th/9112030.

[37] A. A. Tseytlin, "Born-Infeld action, supersymmetry and string theory," arXiv:hep-th/9908105.

[38] A. G. Muslimov, "On The Scalar Field Dynamics In A Spatially Flat Friedman Universe" Class. Quant. Grav. 7, 231 (1990).

D. S. Salopek and J. R. Bond, "Nonlinear Evolution Of Long Wavelength Metric Fluctuations In Inflationary Models", Phys. Rev. D 42, 3936 (1990).

W. H. Kinney, "A Hamilton-Jacobi approach to non-slow-roll inflation" Phys. Rev. D 56, 2002 (1997) [arXiv:hep-ph/9702427].

[39] O. DeWolfe, D. Z. Freedman, S. S. Gubser and A. Karch, "Modeling the fifth dimension with scalars and gravity" Phys. Rev. D 62, 046008 (2000) [arXiv:hepth/9909134].

[40] N. Seiberg and E. Witten, "The D1/D5 system and singular CFT" JHEP 9904, 017 (1999) [arXiv:hep-th/9903224].

[41] A. Fotopoulos and A. A. Tseytlin, "On gravitational couplings in D-brane action" JHEP 0212, 001 (2002) [arXiv:hep-th/0211101]. 
[42] R. Bousso and J. Polchinski, "Quantization of four-form fluxes and dynamical neutralization of the cosmological constant", JHEP 0006, 006 (2000) [arXiv:hep-th/0004134].

[43] C. Armendariz-Picon, T. Damour and V. Mukhanov, "k-inflation" Phys. Lett. B 458, 209 (1999) [arXiv:hep-th/9904075].

[44] T. Chiba, T. Okabe and M. Yamaguchi, "Kinetically driven quintessence" Phys. Rev. D 62, 023511 (2000) [arXiv:astro-ph/9912463].

C. Armendariz-Picon, V. Mukhanov and P. J. Steinhardt, "A dynamical solution to the problem of a small cosmological constant and late-time cosmic acceleration", Phys. Rev. Lett. 85, 4438 (2000) [arXiv:astro-ph/0004134].

C. Armendariz-Picon, V. Mukhanov and P. J. Steinhardt, "Essentials of $k$ essence" Phys. Rev. D 63, 103510 (2001) [arXiv:astro-ph/0006373].

[45] S. Dimopoulos, S. Kachru, N. Kaloper, A. E. Lawrence and E. Silverstein, "Small numbers from tunneling between brane throats" Phys. Rev. D 64, 121702 (2001) [arXiv:hep-th/0104239].

S. Dimopoulos, S. Kachru, N. Kaloper, A. E. Lawrence and E. Silverstein, "Generating small numbers by tunneling in multi-throat compactifications", arXiv:hep-th/0106128. "On The Scalar Field Dynamics In A Spatially Flat Friedman Universe" Class. Quant. Grav. 7, 231 (1990).

D. S. Salopek and J. R. Bond, "Nonlinear Evolution Of Long Wavelength Metric Fluctuations In Inflationary Models", Phys. Rev. D 42, 3936 (1990).

W. H. Kinney, "A Hamilton-Jacobi approach to non-slow-roll inflation" Phys. Rev. D 56, 2002 (1997) [arXiv:hep-ph/9702427].

[46] E. Komatsu et al., "First Year Wilkinson Microwave Anisotropy Probe (WMAP) Observations: Tests of Gaussianity," Astrophys. J. Suppl. 148, 119 (2003) [arXiv:astro-ph/0302223].

[47] V. Acquaviva, N. Bartolo, S. Matarrese and A. Riotto, "Second-order cosmological perturbations from inflation," Nucl. Phys. B 667, 119 (2003) [arXiv:astro-ph/0209156].

[48] J. Maldacena, "Non-Gaussian features of primordial fluctuations in single field inflationary models," JHEP 0305, 013 (2003) [arXiv:astro-ph/0210603].

[49] H. Yavartanoo, work in progress

[50] A. Ghodsi and A. E. Mosaffa, "D-brane dynamics in RR deformation of NS5branes background and tachyon cosmology," arXiv:hep-th/0408015. 